\title{
Perfil de la curva de tolerancia oral a la glucosa en embarazadas sin riesgo de desarrollar diabetes mellitus
}

\author{
Gustavo Gómez Tabarez MD*; Luz Stella Gómez**; Ney Guzmán Gómez***
}

\section{RESUMEN}

OB.IETIVO: Determinar los valores normales de la CTOG en pacientes embarazadas que no presentan factores de riesgo de desarrollar Diabetes Mellitus, en nuestro medio, para tener un punto de referencia en el tamizaje de las pacientes con factores de riesgo.

DISEÑO: Se realizó la curva de glicemia con carga de 100 gramos de glucosa (DEXPAK) por el método de glucosa oxidasa, en suero, tomando muestras en ayunas, una, dos y tres horas después. Se dividieron en grupos de edad gestacional de: 21-24; 25-28; 2932; 33-36; 37-40 semanas de gestación.

LUGAR: El trabajo fue realizado en el departamento de Obstetricia y Ginecología del Hospital Universitario del Valle, con pacientes remitidos de centros periféricos reunían estrictos criterios de inclusión y exclusión

PACIENTES: EI estudio fue realizado finalmente en 337 pacientes en los cuales se define la edad gestacional por FUM confiable y ecografía temprana, asignándose a un grupo determinado aleatoriamente de acuerdo al orden de llegada.

METODOS: Definido la edad gestacional por amenorrea confiable y ecografía se toma muestra en vena antecubital y se centrífuga para obtener el suero donde se determina los niveles de glucosa por el método de glucosa-oxidasa.

RESULTADOS: El 87\% de las pacientes tenían menos de 30 años (X 24 15-42). La mediana, promedio y desviación estándar en ayunas, una hora, dos horas y tres horas fueron $70.8,71.3,8.9 ; 112.8,113.3,27.1 ; 97.0,99.2,22.9$ y $89.8,90.4,20.2$ respectivamente con datos finales de promedio más dos desviaciones estándar de 89.1 en ayunas, 167.5 a la hora, 145 a las dos horas y 130.8 a las tres horas (mgs/dl). Los promedios en ayunas y a las tres horas muestran una significante disminución a medida que avanza el embarazo.

CONCLUSIONES: Se estandariza la curva de tolerancia oral a la glucosa en pacientes sin riesgos de desarrollar diabetes Mellitus en pacientes que consultan al HUV y con carga oral de glucosa de $\mathbf{1 0 0}$ grs por el método de la glucosa oxidasa y se compara con los valores de O'Sullivan encontrándose valores límites de 89, 167, 145 y 131 en ayunas, una, dos, y tres horas respectivamente. Los niveles de glucosa en ayunas y a las tres horas disminuyen a medida que avanza el embarazo. No parece haber diferencias en los valores absolutos pero debido a que son dos técnicas diferentes se concluye que si hay diferencias etnológicas y nutricionales que influyen en el comportamiento metabólico de nuestra población.

PALABRAS CLAVES: Curva de tolerancia oral a la glucosa, diabetes Mellitus, embarazo normal.

\section{SUMMARY}

The objetive of this investigation was to determine the criteria for the glucose oral tolerance test (GTT) in pregnant women without risk of developing Diabetes Mellitus.

We performed glucose oral tolerance test with 100 grs of glucose, and determined serum glucose levels by the glucose oxidase method. Blood samples were taken before breakfast, and one, two, and three hours after. The patientes were grouped by gestational age in $21-24 ; 25-28 ; 29-32 ; 33-36 ; 37-40$ weeks of gestation.

There were 337 patients with reliable diagnosis of gestational age either by LMP or ultrasoud in early pregnancy . The groups were conformed in the orden seen at the outpatient clinics.

$87 \%$ were youngers than 30 years. ( $X 24 ; 15-42$ ). Median, average, and standard deviation fasting, one, two and three hours were: $70.8,71.3,8.9-112.8,113.3,27.1-97.0,99.2,22.9$ y $89.8,90.4,20.2$ respectively. The final data with two SD were 89.1 fasting, 167.5 one hour; 145 two hours, 130.8 three hours.

Average values at fasting and three hours were significantly low while duration of pregnancy In conclusion we standarized the glucose oral tolerance test (GTT) in pregnant women without risk of developing Diabetes Mellitus by oral ingestion of 100 grs of glucose and blood sugar determinations by the glucose-oxidase method. Comparison with the O'Sullivan curve values were made.

The limit curve values were: $89,167,145,131$ fasting, one, two and three hours respectively There not absolut values diferences with the O'Sullivan curve values eventhough the techniques. We believe that etnic and nutritional diferences are reflected in the metabolism of the population thah we studied.

KEY WORDS: Oral glucose tolerance test, diabetes mellitus, normal pregnancy.

\footnotetext{
* Profesor Titular. Departamento de Obstetricia y Ginecología. Sección de Endocrinología e Infertilidad. Universidad del Valle.

** Bacterióloga. Departamento de Obstetricia y Ginecología. Laboratorio de Endocrinología e Infertilidad. Hospital Universitario del Valle.

*** Profesor Titular (J). Departamento de Medicina Social. Universidad del Valle.
} 


\section{Introducción}

Los valores de la curva de tolerancia a la glucosa en mujeres embarazadas continúa un tema de mucha controversia. Se acepta generalmente que el embarazo disminuye la sensibilidad a la insulina exógena (1), produce una degradación placentaria aumentada (2) y conduce a miles altas de actividad parecidas a la insulina (3). Con respecto a la respuesta obtenida con la administración oral se han notado cambios aún en presencia de pruebas intravenosas normales de tolerancia a la glucosa (4-6). Algunos investigadores aconsejan precaución en la interpretación de pruebas orales de tolerancia a la glucosa, debido a que los resultados pueden estar influenciados por cambios en la absorción intestinal de glucosa (7).

Sin mucha controversia y sin estudios de validación para características inherentes y adquiridas en altas latitudes se han venido aceptando, en la práctica médica los criterios de O'sullivan y Mahan, presentada en la 23 reunión anual de la Asociación Americana de Diabetes realizada en Atlanta en Junio de 1963 (8). Estos investigadores realizaron un estudio con 752 mujeres, a la cuales les administraron 100 gramos de glucosa oral a distintas edades gestacionales. La distribución de los resultados de glicemia por el método de Somogy-Nelson permitieron establecer tres niveles según estados o grados de severidad.

Los criterios derivados se aplicaron entonces a los resultados de pruebas de glicemia en 1.013 mujeres, seleccionadas según edad gestacional. Una fuerte correlación se encontró entre la severidad de las clasificaciones originales y los porcentajes dentro de cada grupo que posteriormente desarrollaron diabetes (test nivel $\mathrm{Y}$ solamente el $6.9 \%$, test de nivel II solamente $16.1 \%$ y test de nivel III solamente $4(0.3 \%)$.

El criterio considerado apropiado para la aplicación rutinaria consiste de dos o más de los siguientes valores iguales o excedidos: ayuno $90 \mathrm{mg}$ por $100 \mathrm{ml}$; una hora $165 \mathrm{mg}$ por $100 \mathrm{ml}$, dos horas $145 \mathrm{mg}$ por $100 \mathrm{ml}$, tres horas $125 \mathrm{mg}$ por $100 \mathrm{ml}$.

El presente estudio repite la metodología de O'sullivan y Mahan, con mujeres de distinta etnicidad y edades gestacionales, distribución geográfica y además con la técnica de la glucosa oxidasa para determinación de los valores de glicemia.

El estudio busca reafirmar los criterios de O'sullivan y Mahan para mujeres colombianas y en general para latinoamericanas.

\section{Material y métodos}

Población de estudio: Se seleccionaron 337 mujeres que asistían a la consulta prenatal de la consulta externa del Hospital Universitario "Evaristo García" de la ciudad de Cali. Se excluyeron mujeres en el primer trimestre de embarazo ( $£ 13$ semanas de gestación) y en el segundo trimestre (14-27 semanas de gestación), debido al número pequeño que impedía un análisis comparativo con el grueso de mujeres que estaban en el tercer trimestre de embarazo (28-40 semanas de gestación). Igualmente se excluyeron mujeres embarazadas con antecedentes de diabetes, feto grande, anomalías congénitas, toxemias e historia de feto muerto, muerte neonatal y aquellas cuyo embarazo terminó con recién nacido mayor de 3.500 gramos de peso.

\section{Resultados}

Distribución por edad. E1 87.5\% de las mujeres embarazadas a quienes se les practicó la prueba de tolerancia a la glucosa, tenían menos de 30 años de edad. La cuarta parte estaban entre 15 y 19 años, y apenas cuatro de ellas tenian 40 y más años de edad. Los extremos del rango fueron 15 y 42 años, con un promedio de edad de 24, una media de 23 y lina desviación standard de 5.4 (tabla 1).

Valores de las pruebas de tolerancia a la glucosa. Obsérvese en la tabla 2 los valores de la media; promedio y desviación standar de las pruebas de determinación de glicemia en ayunas, una hora, dos horas y tres horas después.

El test se considera positivo si dos o más lecturas iguala o excede los valores especificados en el cuadro. El criterio de tamizaje preferido es el promedio más dos desviaciones estándares.

Tabla 1

DISTRIBUCION POR GRUPOS DE EDAD DE MUJERES EN TERCER TRIMESTRE DE EMBARAZO

\begin{tabular}{|lll|}
\hline Edad & No. & $\%$ \\
\hline $15-19$ & 87 & 25.82 \\
$20-24$ & 129 & 38.28 \\
$25-29$ & 79 & 23.44 \\
$30-34$ & 27 & 8.01 \\
$35-39$ & 11 & 3.26 \\
$>=40$ & 4 & 1.19 \\
\hline TOTAL & $\mathbf{3 3 7}$ & $\mathbf{1 0 0 . 0 0}$ \\
\hline
\end{tabular}

Los valores según criterios de O'sullivan y Mahan por miles son:

\begin{tabular}{|lllll|}
\hline & Ayuno & 1 Hora & 2H & 3H. \\
\hline Test Nivel I (promedio + 1 D.E) * & 80.0 & 140.0 & 120.0 & 110.0 \\
Test Nivel II (promedio +2 D.E)* & 89.0 & 167.0 & 145.0 & 130.0 \\
Test Nivel III (promedio +3 D.E)* & 98.0 & 194.0 & 168.0 & 151.0 \\
\hline
\end{tabular}


Tabla 2

RESULTADOS DEL TEST DE TOLERANCIA A LA GLUCOSA

\begin{tabular}{|lllll|}
\hline Medidas & Ayuno & 1 hora & 2 horas & 3 horas \\
\hline Mediana & 70.8 & 112.8 & 97.0 & 89.8 \\
Promedio & 71.3 & 113.3 & 99.2 & 90.4 \\
Desv. Standar & 8.9 & 27.1 & 22.9 & 20.2 \\
\hline
\end{tabular}

Valores en mgs por $100 \mathrm{ml}$

TOLERANCIAA LA GLUCOSA: Obsérvese en la tabla 3

La distribución de los valores de tolerancia a la glucosa en ayunas y a intervalos de una hora. Nótese la configuración de los histogramas y polígonos de frecuencia que siguen con bastante precisión una distribución Gaussiana, con excepción de la glicemia en algunas que es mas picuda. ( Fig 1.)

Esta distribución de "normalidad" apoya el uso de los promedios y desviaciones standares que se describen la mayor dispersión de los datos.

Valores de tolerancia a la glucosa según semanas de gestación

En la tabla 4 se observan los valores de las medianas, promedios y desviaciones standares de las 337 mujeres embarazadas según edad gestacional al momento de determinar las glicemias. Nótese la disminución de los promedios

* Frutrade Significancia

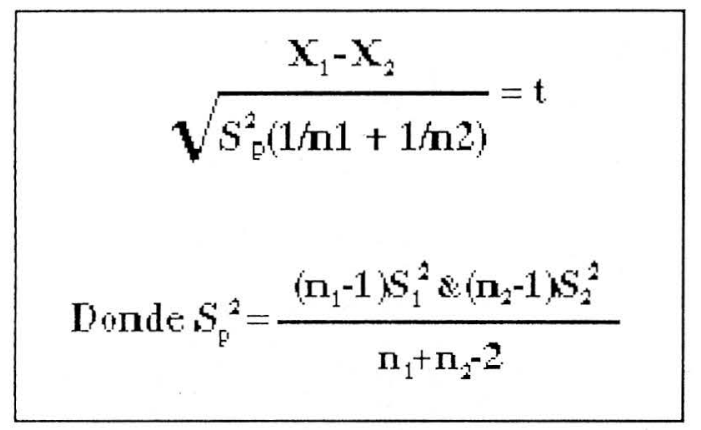

a medida que aumenta la edad gestacional, diferencia de promedios que fue estadísticamente significante para el primer grupo (28-31 semanas), comparado con el tercer grupo ( $>=36$ semanas) con una $\mathrm{P}<0.005$. No hubo diferencias estadísticamente significantes para las glicemias a intervalos de tiempo.

\section{Discusión}

La clínica de Embarazo de alto riesgo del Hospital Universitario del Valle es un sitio de referencia de atención obstétrica de mayor complejidad. El 1\% de las pacientes atendidas tienen diagnostico de Diabetes Mellitus. La mortalidad perinatal ( fetal tardía y neonatal temprana) es del $79 \mathrm{x}$ mil nacidos vivos mayores de $999 \mathrm{~g}$ versus $45 \mathrm{x}$ mil en la población obstétrica no diabética con una incidencia de cesáreas de 38.9 vs 26.1, mayor morbilidad materna (infección urinaria, preeclampsia e hipertensión arterial previa) $32.7 \%$ vs $26.8 \%$ y mayor morbilidad fetal (macrosomía, SDR, defectos congénitos ) $35.5 \%$ vs $14.8 \%$ respectivamente (9).

El énfasis en la detección temprana durante el embarazo y la remisión de la paciente una vez hecho el diagnostico, la normalización de los niveles de glucosa en las pacientes conocidas como diabéticas antes de embarazarse, han sido los mecanismos invocados para disminuir morbi y mortalidad fetal en nuestro medio en particular (10-11).

Indiscutiblemente la detección temprana de la hiperglicemia en el embarazo es muy importante para la disminución de la morbimortalidad fetal. Por otra parte, aunque la mortalidad materna es rara, la morbilidad materna está incrementada significativamente (11-12).

Se ha descrito que la hiperglicemia alrededor de la 5 semana de gestación es la responsable de la aparición de malformaciones congénitas (13). La hiperglicemia por su parte va a ser causa de macrosomía, con la patología obstétrica que ésta genera, retardo en la maduración pulmonar, aumento del SDR, muerte súbita intrauterina, hipocalcemia e hipoglicemia neonatal (14-16).

Tabla 3

DISTRIBUCION DE LOS VALORES DE TOLERANCIA A LA GLUCOSA

\begin{tabular}{|lllllllll|}
\hline \multirow{2}{*}{ Glicemia } & \multicolumn{2}{c}{ Ayuno } & \multicolumn{2}{c}{ 1 Hora } & \multicolumn{2}{c|}{ 2 Horas } & \multicolumn{2}{c|}{ 3 Horas } \\
& No & \% & No. & \% & No. & \% & No. & \% \\
\hline $40-59$ & 0 & 0 & 3 & 0.9 & 4 & 1.2 & 2 & 0.6 \\
$60-79$ & 36 & 10.7 & 4 & 1.2 & 6 & 1.8 & 16 & 4.8 \\
$80-99$ & 260 & 77.1 & 27 & 8.0 & 40 & 11.9 & 88 & 26.1 \\
$100-119$ & 40 & 11.9 & 84 & 24.9 & 139 & 41.2 & 146 & 43.3 \\
$120-139$ & 1 & 0.3 & 98 & 29.1 & 97 & 28.8 & 63 & 18.7 \\
$140-159$ & 0 & 0 & 74 & 22.0 & 39 & 11.6 & 19 & 5.6 \\
$160-179$ & 0 & 0 & 29 & 8.6 & 8 & 2.3 & 1 & 0.3 \\
$>=180$ & 0 & 0 & 18 & 5.3 & 4 & 1.2 & 2 & 0.6 \\
TODOS & 337 & 100 & 337 & 100 & 337 & 100 & 337 & 100 \\
\hline
\end{tabular}

* Las cifras han sido redondeadas para facilitar las clasificaciones. 
Tabla 4

DISTRIBUCION DE LOS VALORES PROMEDIOS

MEDIANA Y DESVIACION ESTANDARES DE 337

MUJERES EMBARAZADAS, SEGUN SEMANAS DE GESTACION

\begin{tabular}{|lcrrr|}
\hline $\begin{array}{l}\text { Semanas de } \\
\text { gestación }\end{array}$ & Ayuno & \multicolumn{3}{c|}{$\begin{array}{c}\text { Valores de } \\
\text { tolerancia }\end{array}$} \\
\hline $\begin{array}{l}\text { 28-31 (n=96) } \\
\text { Mediana }\end{array}$ & 73.0 & 110.0 & 99.0 & 94.0 \\
$\begin{array}{l}\text { Promedio } \\
\text { Desv Stándar }\end{array}$ & $74.0 *$ & 113.0 & 100.0 & 93.0 \\
\hline 32-35 (n=118) & 8.5 & 28.8 & 23.8 & 19.2 \\
Mediana & 70.0 & 110.0 & 96.0 & 92.0 \\
Promedio & 71.0 & 114.0 & 100.0 & 90.0 \\
Desv Stándar & 9.7 & 29.4 & 23.1 & 22.5 \\
\hline $\begin{array}{l}\text { 336 (n=123) } \\
\text { Mediana }\end{array}$ & 68.7 & 114.0 & 97.0 & 89.0 \\
Promedio & $70.0 *$ & 114.0 & 98.0 & 88.0 \\
Desv Stándar & 23.5 & 23.5 & 22.2 & 18.5 \\
\hline
\end{tabular}

Diferencia de Promedios \pm e.e

Entre los métodos de tamizaje la curva de tolerancia oral a la glucosa es la mas ampliamente aceptada. Sin embargo, todavía hay controversia acerca de método usado, si oral o venosa ; cantidad de glucosa usada e interpretación de límites para determinar anormalidad (17).

El presente estudio estandariza y propone valores relacionados con la técnica usada en sangre venosa después de una carga de 100 gramos de glucosa por vía oral en pacientes nativas, comparada con los métodos usados en un grupo diferente étnico, cultural y nutricionalmente (18-20).

$\mathrm{Si}$ escogemos como nivel de tamizaje los valores promedio de glicemia ( $\mathrm{mg}$ por $100 \mathrm{ml}$ ), más dos desviaciones estándar los resultados del presente estudio no difiere grandemente de aquellos encontrados por O'Sullivan (tabla 5).

Según este criterio todas las mujeres embarazadas con glicemias iguales o por encima de estos valores deberían considerarse con una alta probabilidad de tener o desarrollar posteriormente una diabetes mellitus.

Tabla 5

VALORES DEL TEST DE TOLERANCIA A LA GLUCOSA, EN MUJERES EMBARAZADAS

\begin{tabular}{|l|ll|}
\hline Tolerancia & \multicolumn{2}{|c|}{ Glicemia en $\mathbf{~ m g ~ X ~} \mathbf{1 0 0} \mathbf{~ m l}$} \\
\hline & O'Sullivan & Gómez \\
\hline Ayuno & 90 & 89 \\
1 Hora & 165 & 167 \\
2 Horas & 145 & 145 \\
3 Horas & 125 & 130 \\
\hline
\end{tabular}

Figura 1

DISTRIBUCION DE LOS VALORES DE TOLERANCIA A LA GLUCOSA



Figura 1

DISTRIBUCION DE LOS VALORES DE TOLERANCIA A LA GLUCOSA

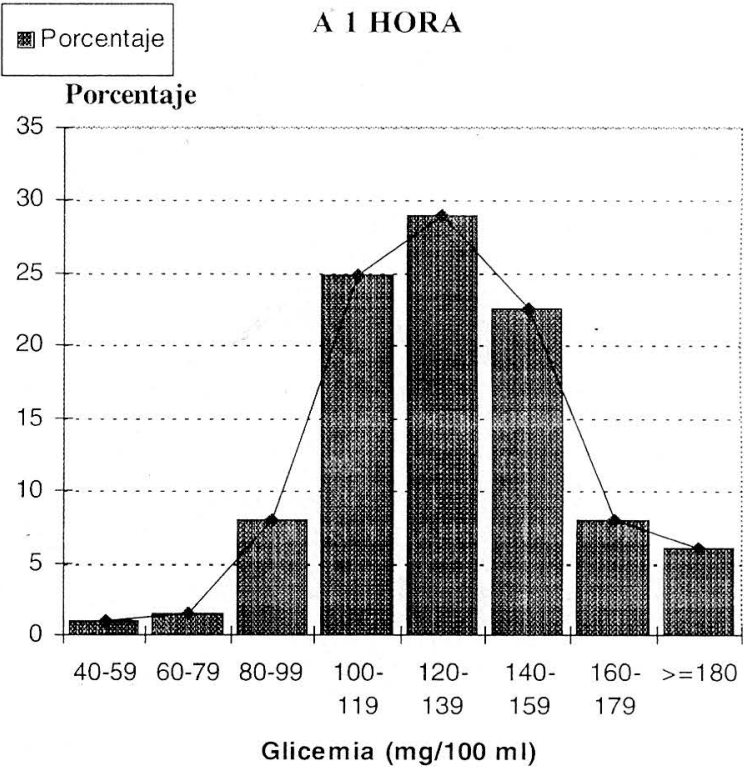

Sin embargo, del mismo estudio de O'sullivan citado anteriormente se observa que apenas un $16.1 \%$ de las mujeres encontradas en este rango promedio + dos desviaciones estándar, desarrollaron posteriormente diabetes y que el porcentaje fue mayor (40.3\%), cuando los valores igualaron o excedieron el promedio más tres desviaciones estándar. Hubo, también un $6.9 \%$ de mujeres que desarrollaron diabetes con un test que igualó o superó el promedio más una desviación standar.

Esto, por supuesto refleja la superposición de dos poblaciones. La población tamizado está compuesta por un grupo de mujeres "enfermas" (con diabetes mellitus) y un 
Figura 1

DISTRIBUCION DE LOS VALORES DE TOLERANCIA A LA GLUCOSA

\section{A 2 HORAS}

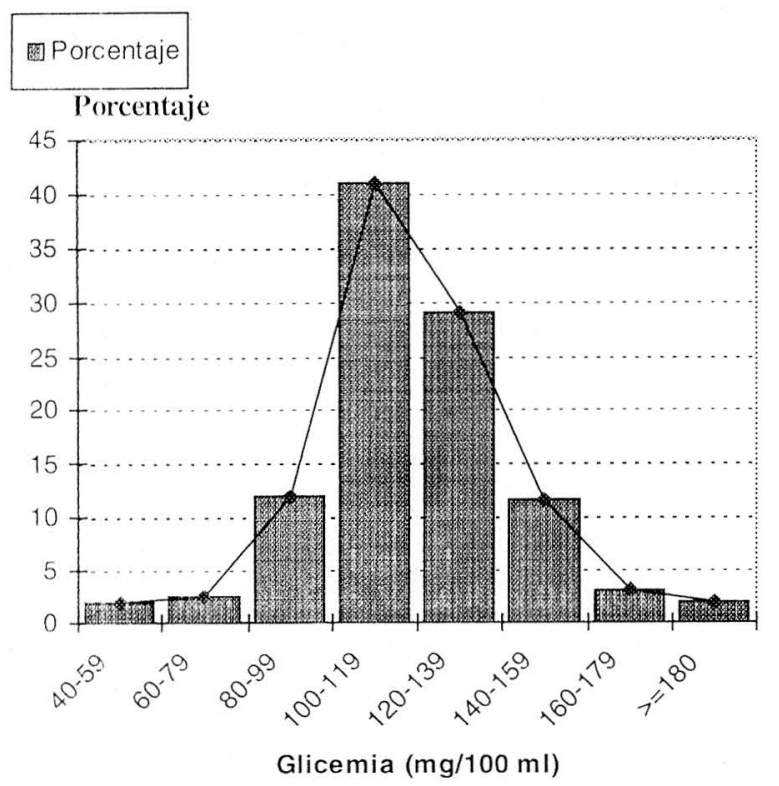

grupo de mujeres no enfermas, ambas poseyendo los valores de tolerancia a la glucosa, a varios niveles de medición Para algunos valores las distribuciones de estas dos poblaciones se superponen y nos es posible asignar mujeres embarazadas a ninguno de los grupos de "normalidad" o presumiblemente "enfermas". Aumentar el nivel de tamizaje por ejemplo a valores iguales al promedio más tres desviaciones estándar mejoraría grandemente la sensibilidad pero con una merma considerable de la especificidad.

La prueba de tamizaje más satisfactoria sería la que mida la glicemia una o dos horas después de una carga estandarizada de glucosa.
Figura 1

DISTRIBUCION DE LOS VALORES DE TOLERANCIA A LA GLUCOSA

\section{A 3 HORAS}

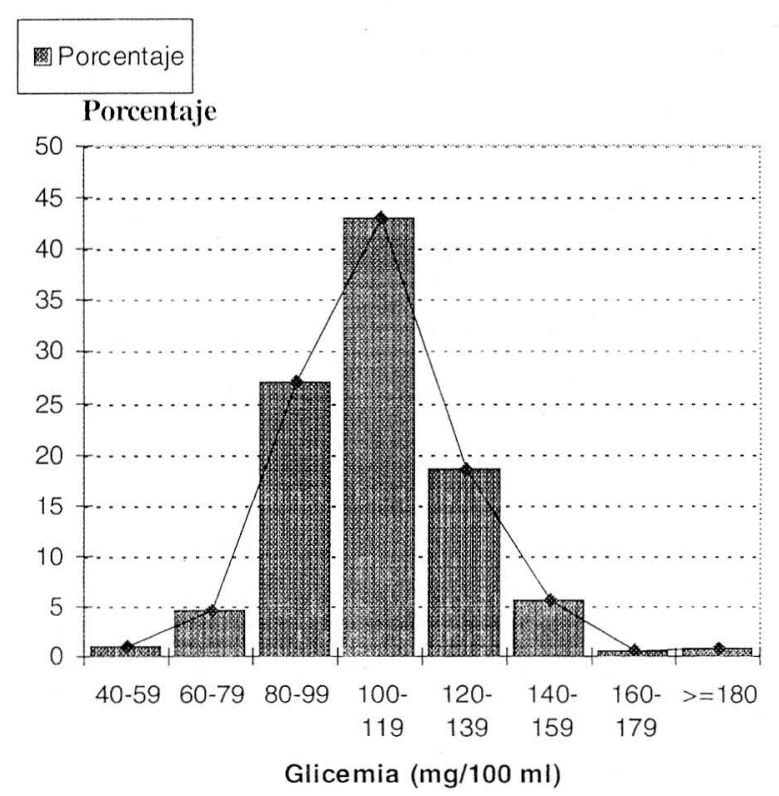

De otro lado los valores obtenidos en el presente estudio pueden utilizarse como criterio de "normalidad" (no enfermedad), cuando la glicemia determinada en mujeres embarazadas está por debajo de los promedios en especial del nivel II.

Como corolario a la presente investigación deberá decirse que dado el estado de arte de las pruebas de tamizaje para diabetes mellitus en mujeres embarazadas si se quiere mejorar la precisión del test debe aumentarse los niveles de tamizaje y complementarse con una buena historia clinica que explore antecedentes familiares y consecuencias de la enfermedad en embarazos anteriores y el presente.

\section{BIBLIOGRAFIA}

1. Burt RL. Peripheral utilization off glucose in pregnancy III insulin tolerance, Obstet. Gynec 1956; 7: 658.

2. Freinkel, N. and Goodner, CJ. Carbohydrate metabolism in pregnancy. Y. The metabolism of insulin by human placental tissue. J. Clin Invest. 1960; 39: 116-131.

3. Spellacy WN. and Goetz FD. Plasma insulin levels in pregnancy. Clin Research 1962; 10: 299.

4. Hurwitz D. and Jensen D. Carbohydrate metabolism in normal pregnancy. New. Eng. J. Med. 1946; 234: 327-329.

5. Cobley J.F.C.C and Lancester HO. Carbohydrate tolerance in pregnancy. Med. J. Aust. 1955; 1: 171-175.

6. Welsh WW. 3er Studies of abnormal glucose metabolism in pregnancy. Diabetes 1960; 9: 466-470.

7. Burt RL. Glucose tolerance in pregnancy. Diabetes II: 1962; 227-229.

8. O'Sullivan IB and Mahan. Critera for the oral glucose tolerance test in pregnancy. Diabetes, 1964; 13: 278-285.
9. Ortiz I. Sistema informativo perinatal CLAP-OPS/OMS departamento OB-GIN U del Valle. Comunicación personal mayo 1997.

10. Gómez G. Diabetes y embarazo. Rev. Col. Obstet. Ginecol. 1990; $41: 81$.

11. Gómez G., Rebolledo A., Restrepo C. Morbimortalidad fetal por diabetes en la Clínica de Embarazo de Alto Riesgo del Hospital Universitario del Valle Colombia Médica 1991; 22: 144.

12. Gabbe SG., Mestman JH., Freeman RK et al. Management and outcoe of pregnancy in diabetes Mellitis class B to R. Am. J. Obstet. Gynecol.

13. Jovanovic PL., Peterson CM. Abnormal metabolism and the risk for birth defects with emphasis in diabetes. Ann NY Acad Science 1993; 678: 228 .

14. Jacobson JD., Cousin LA. A population -based study of maternal and perinatal outcome in patients with gestational diabetes. Am. J. Obstet. Gynecol. 1989; 161:981. 
15. Pallardo LF., Grande C., Luna R., Megia A., GonzálezA. Diabetes y embarazo. Nuestra experiencia en diabetes gestacional. Med. Clin. (Barc) 1990; 95: 406.

16. Salvens D̂R., Brudenell JM., ProdlerAJ., et al, Fetal pancreatic beta-cell,function in pregnancy complicated by maternal diabetes mellitus,and tits relationship with fetal acidemia and macrosomia. Am. J. Obstet. Gynecol. 1993; 168: 1363.-9

17. Gómez G. Diabetes y Embarazo. En Obstetricia de Alto Riesgo. Cifuentes (ed). XYZ impresores, 1994; 619.
18. Cousin L., Baxi L., Chez R et al. Screening recommendation for gestational diabetes mellitus.Am. J. Obstet. Gynecol. 1991; 165: 493.

19. Hare JW., White P. Gestational diabetes and the white clasification. Diabetes Care 1980; 3: 394.

20. Arias F. Diabetes and pregnancy. In Practical guide to HighRisk pregnancy and delivery. Arias F (ed). Mosby Year Book, $1993 ; 280$. 